\title{
Wie fliegen wir morgen? \\ - Neue Tendenzen im Luftverkehr
}

\author{
Thomas Biermann
}

\section{Zusammenfassung}

Der Fortschritt im Luftverkehr wird eher in kleinen Schritten erfolgen, denn Verkehrsflugzeuge stellen eine reife Technologie da, die kaum mehr revolutionäre Neukonstruktionen erwarten lässt. Aber auf längere Sicht können die zunehmende Individualisierung und Automatisierung dramatische Veränderungen der Flugreise mit sich bringen.

\begin{abstract}
Progress in air transport will be incremental, as building commercial airliners has become a mature technology, where revolutionary new designs should not be expected in the foreseeable future. But individualisation and automatisation could in the long run lead to dramatic changes in the way we travel by air.
\end{abstract}

\section{Lufttransport heute}

\subsection{Das Jahrhundert des Flugzeugs}

Das 21. Jahrhundert ist das Jahrhundert des Flugzeugs, so wie das 19. Jahrhundert die Ära der Eisenbahn und das 20. Jahrhundert die des Automobils war. Der Flughafen, nicht mehr der Hauptbahnhof ist heute Mittelpunkt der Verkehrsströme und zentraler Erfolgsfaktor der regionalen Entwicklung (Thompson 2007). In der Weltwirtschaft dient der Luftverkehr zunehmend auch dem Gütertransport - zwar wird nur 1\% der Gütertonnage im weltweiten Außenhandel per Luftfracht abgewickelt, doch macht dies, weil primär die höherwertigen Produkte den Luftweg nehmen, fast $40 \%$ des gesamten Warenwertes aus (Tretheway 2007).

\subsection{Das Verkehrsflugzeug ist ausgereift}

Eine Revolution des Luftverkehrs durch vollkommen neue Antriebe oder Materialien ist für die kommenden 20 Jahre praktisch auszuschließen. Der Motorflug ist nunmehr gut 100 Jahre alt und demnach eine reife Technologie, welche die ganz großen Sprünge der Entwicklung hinter sich hat.

Die Leistungsfähigkeit des Flugzeuges hinsichtlich Nutzlast und Reichweite nimmt weiter zu, entgegen früherer Erwartung wird sich indes in der Dimension Geschwindigkeit - typischerweise im Bereich um 800 $\mathrm{km} / \mathrm{h}$ - in einem absehbaren Zeithorizont nichts ändern. Überschallgeschwindigkeit ist nicht allein aus wirtschaftlichen Erwägungen kein Thema mehr, die Sensibilität in Umweltfragen lässt derartig lärmintensive und energiefressende Technologien nicht mehr zu.

Unter Experten noch in der Diskussion ist das NurFlügel-Flugzeug. Die Integration von Zelle und Flügel hätte aus Sicht des Passagiers »fliegende Kinosäle« mit ei- ner ganz anderen Innenraumstruktur mit sich gebracht, doch zeigen sich bei diesen futuristischen Konzepten bislang technisch kaum beherrschbare Probleme. Es steht demnach zu erwarten, dass die großen Verkehrsflugzeuge ihre seit Einführung der Jets in den späten 1950er Jahren vertraute Silhouette für die nächsten Jahrzehnte beibehalten. Der Fortschritt findet - wie beim Auto - vorwiegend »unter der Haube«statt.

\subsection{Vorwärts in kleinen Schritten}

Aber er findet statt: die Zellenstruktur der neuen Boeing $787 »$ Dreamliner « besteht beispielsweise weitgehend aus Kunststoff statt Aluminium. Die Gewichtsersparnis reduziert den Treibstoffverbrauch und damit die Umweltbelastung. Der Passagier profitiert direkt von dem Wandel der Werkstoffe. Die für das Wohlbefinden so belastende Trockenheit der Luft an Bord eines Verkehrsflugzeuges beruht nämlich darauf, dass die Klimaanlagen entsprechend eingestellt sind, um die Korrosion der Metallstruktur zu vermeiden. In einem »Plastikflugzeug « kann hingegen normale Luftfeuchtigkeit herrschen.

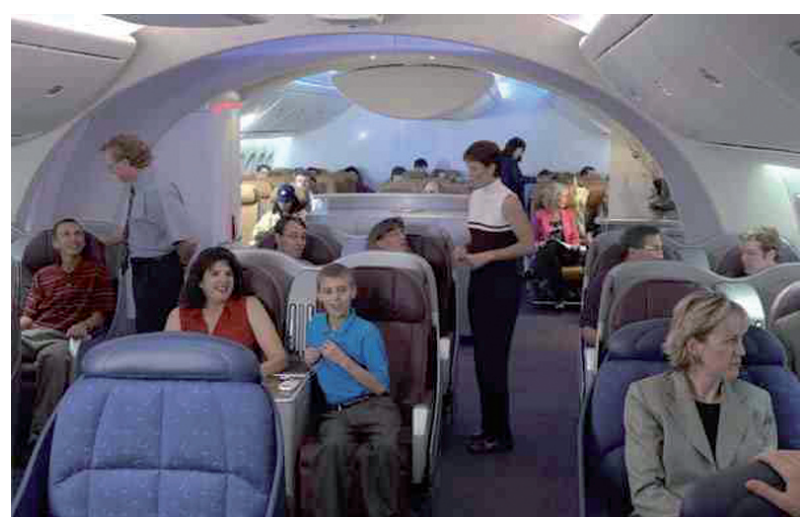

Abb. 1: Boeing 787 (Foto: Boeing) 
Auf kurze Sicht definieren viele kleine Veränderungen wie die oben geschilderte den Fortschritt:

- kostengünstige Langstreckenflugzeuge im 200-Sitzer Format erlauben Direktverbindungen, wo bislang die Reise nur per Umsteigen realisierbar war;

- Telefon und Internet an Bord werden bald selbstverständlich sein;

- der Schlafkomfort in den First Class-Sitzbetten ist bald höher als bei der Mehrzahl der Menschen zu Hause;

- die Automatisierung von Reservierung und Ticketing erlaubt Selbstbedienungskonzepte, wo früher viel menschliche Arbeitskraft notwendig war;

- optimierte Bodenprozesse erhöhen die Produktivität von Flugzeug und Crew, sie erlauben Billigflüge, die dem Luftverkehr neue Zielgruppen erschließen (Biermann 2005).

\section{Neue Trends}

\subsection{Individualisierung in der Luft}

Seit mehreren Jahren erleben die Business Jets einen ungeheuren Aufschwung, gegenwärtig sind nach Expertenschätzungen weltweit 11.000 Einheiten im Einsatz, davon $75 \%$ in den USA. Die Vorteile dieser Maschinen sind vielfältig. Die Unabhängigkeit von Flugplänen und die Vermeidung zeitraubender Sicherheitskontrollen am Flughafen spielen eine wichtige Rolle. Der Hauptvorteil besteht jedoch in der Möglichkeit, Orte abseits der wesentlichen kommerziellen Flugrouten - etwa in Osteuropa oder der chinesischen Provinz - direkt zu erreichen (Economist 2007).

Die Anfangsinvestition für ein modernes Geschäftsreiseflugzeug liegt allerdings im Bereich von knapp 10 Mio. € für einen 7-Sitzer Lear Jet und bis zu 50 Mio. € für das Spitzenmodell Bombardier Global Express, das 19 Passagiere über $11.000 \mathrm{~km}$ weit befördern kann. Die Betriebskosten für zwei Piloten, Wartung, Treibstoff, Landegebühren etc. übersteigen schnell die 1 Mio. €-Grenze pro Jahr.

Daher operieren viele solcher Jets im Teileigentum. Für einen Bruchteil des Kaufpreises erwirbt man ein Nutzungsrecht für beispielsweise $25 \%$ der verfügbaren Zeit. Nach Expertenschätzungen wird ein für die Eigennutzung angeschafftes Geschäftsreiseflugzeug im Durchschnitt nur 325 Stunden im Jahr geflogen, eine Maschine in Fractional Ownership hingegen 900 Stunden, wodurch sich eine wesentlich günstigere Verteilung der Fixkosten ergibt (Singh 2006).

Der individualisierte Luftverkehr wächst jedes Jahr doppelt so schnell wie der Gesamtmarkt. Er dürfte in Zukunft weiteres Potenzial durch die neue Kategorie der Very Light Jets (VLS) gewinnen, die am Beginn der Serienproduktion stehen. Dabei handelt es sich um extrem leicht gebaute kleine Flieger, die 3 bis 6 Passagiere befördern und die Fähigkeit besitzen, auf Bahnen von nur 900 m Länge zu starten und zu landen. Sie sind für Stückpreise ab 1,6 Mio. € zu haben und können von einem statt zwei Piloten geflogen werden. Ein Indikator für die Wachstumsaussichten in diesem Segment ist die Tatsache, dass nicht nur kleine Startup-Unternehmen ihr Glück mit der Entwicklung von VLJs versuchen, sogar der japanische Automobilhersteller Honda hat ein serienfertiges Modell vorgestellt.

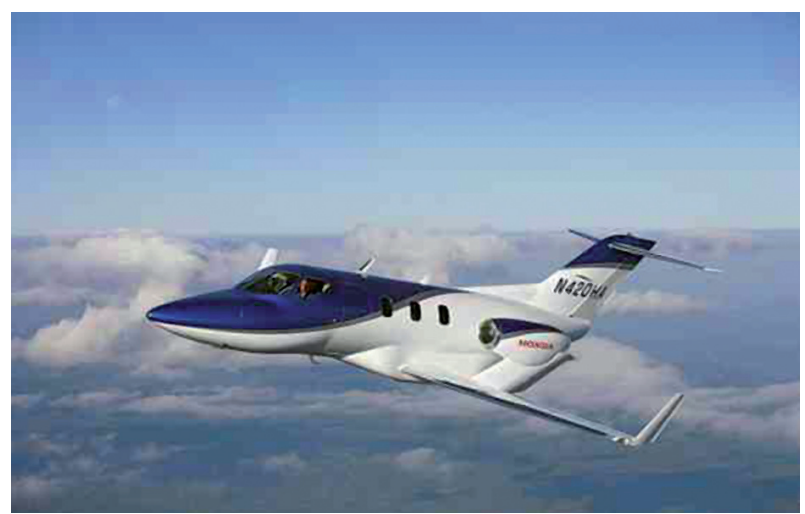

Abb. 2: Hondajet (Foto: Honda)

Der Investitionsbedarf und die Betriebskosten fallen um Größenordnungen günstiger aus als bei herkömmlichen Jets. Als magische Kostengrenze für die Massennutzung gilt der Wert von 1 US-Dollar pro Meile und Passagier (grob gerechnet 50 Eurocents pro PassagierKilometer), womit ein VLJ-Flug dem Preisrahmen eines Linienfluges in First-/Business-Class vergleichbar wäre (Singh 2006). Das eigene Flugzeug, die Beteiligung an einem Timesharing-Modell oder die Nutzung von LuftTaxi-Diensten wird so selbst für das Management eines mittelständischen Unternehmens mit entsprechenden Reisebedürfnissen überlegenswert. Die Firma Day Jet in Florida hat 240 VLJs vom Typ Eclipse 500 als Luft-Taxi geordert, die ersten drei Exemplare wurden im März 2007 geliefert.

\subsection{Automatisierung am Boden und an Bord}

Als zweites Thema mit hohem Aufmerksamkeitswert gilt die Automatisierung, insbesondere im Cockpit der Verkehrsflugzeuge. Heute steuern zwei Männer (manchmal auch Frauen) einen Airbus oder eine Boeing. Früher waren auf einem Flugdeck fünf Leute beschäftigt. Die Fortentwicklung der Elektronik machte den Funker und den Navigator bald überflüssig, der dritte Mann - der Flugingenieur - verschwand ab 1980. Steht nun der Copilot zur Disposition, vielleicht gar der Kapitän?

Auf die Flugdurchführung spezialisierte moderne Bordcomputer sind längst in der Lage, das Flugzeug allein zu fliegen. Die Besatzung leistet auf vielen Flügen nicht viel mehr als den Druck auf den Startknopf und eine Lautsprechdurchsage. Menschliche Piloten sind in unvorhergesehenen Sondersituationen zweifellos wertvoll, doch ist die Technik mittlerweile so zuverlässig, dass solche Situationen kaum noch vorkommen. Menschen bringen auf der anderen Seite selbst viele Probleme mit ein - sie machen Fehler, werden krank, streiken oder stecken auf dem Weg zum Airport im Stau.

Piloten sind außerdem teuer. Dafür ist neben der aufwändigen Ausbildung und dem Gehalt bei geringer 
Produktivität (Flugstundenbegrenzung wegen strikten Ruhezeitregeln) die Technik mit verantwortlich. Ein modernes Cockpit erfordert extrem kostspielige MenschMaschine-Schnittstellen, weil ein elektrisches Warnsignal vom Radar nicht direkt an die Steuerung geht, sondern über das Auge und die Hand des Flugzeugführers geleitet werden muss.

Unbemannte Flugzeuge befinden sich daher im Aufwind. Im militärischen Bereich sind Aufklärungsdrohnen und unbemannte Kampfjets bereits seit den 1980er Jahren Routine. Im zivilen Bereich gibt es nach einer Übersicht der NASA mittlerweile ebenfalls eine große Zahl von Anwendungen von der Fischereiüberwachung über den Agrarflug - Ausbringung von Düngern und Pestiziden bei großen Landwirtschaftsflächen - bis zu fliegenden Mobilfunkantennen (Cox et. al. 2004).

Das Hauptargument für unbemanntes Fliegen liegt im zivilen Sektor in der potenziell sehr langen Verweildauer in der Luft. Da der schwere Pilot und die noch schwereren Lebenserhaltungs- und Steuersysteme eingespart werden, kann mehr Treibstoff an Bord mitgeführt werden. Aus diesem Grund bleibt das Gerät mühelos bis zu 40 Stunden in Aktion - und so ein Autopilot wird auch nicht müde.

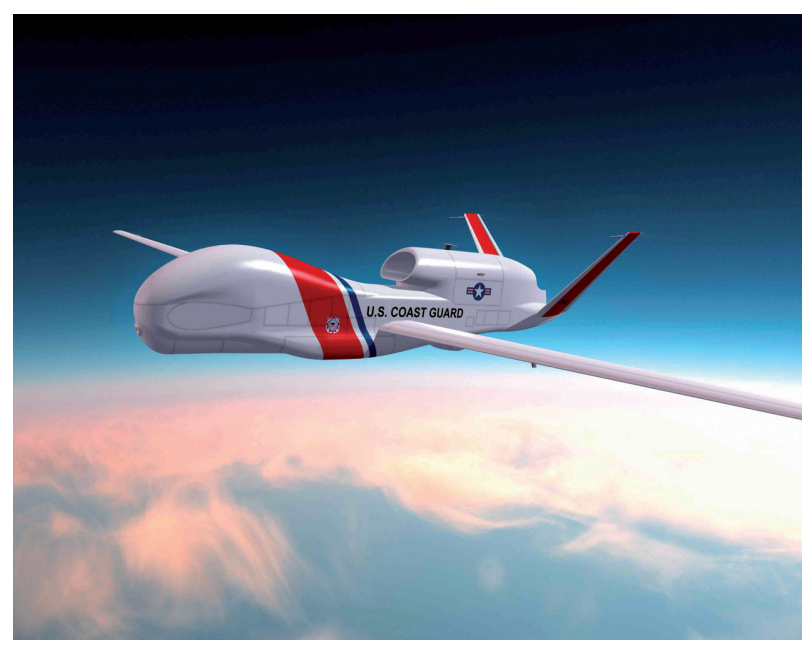

Abb. 3: Global Hawk (Foto: Northrop Grumman)

Die regelmäßig eingesetzten unbemannten Fluggeräte sind gegenwärtig noch recht klein, ihre Größe reicht vom gehobenen Spielzeugformat bis zum Kleinflugzeug. Diese so genannten UAVs (unmanned aerial vehicles) bewegen sich

- autonom und fliegen ein vorher definiertes Programm $\mathrm{ab}$, oder

- werden vom Boden gesteuert, wobei der Leitstand mehrere solcher Flugkörper gleichzeitig im Auge halten kann, oder

- sie werden durch ein bemanntes »Mutter-Flugzeug « kontrolliert, das aus der Luft in Sichtnähe ein UAVRudel steuert.

Mit hoher Wahrscheinlichkeit gehört die Zukunft autonom operierenden UAVs, bei denen ein Bodenleitstand nur im Bedarfsfall »remote« eingreift.

Ab 2020 ist mit deutlich größeren unbemannten Transportfliegern zu rechnen, vor allem für routinemä- ßig anfallende kleinere Sendungen abseits der Hauptverkehrsströme im Kurier- und Expressverkehr (Cox et. al. 2004). Im Passagierverkehr hingegen wird das pilotenlose Flugzeug auf sich warten lassen. Komplizierte Rechtsund Haftungsprobleme sind noch zu lösen und die Akzeptanz des Publikums ist ungewiss. Im Schienenverkehr gibt es bereits positive Erfahrungen mit führerlosen Fahrzeugen im Nahverkehr, insbesondere auf Flughäfen zwischen weit entfernten Terminals und auf einzelnen U-Bahn-Strecken. Für die Mehrzahl der Menschen dürfte aber der Einstieg in ein Flugzeug ohne menschlichen Kommandanten heute kaum zumutbar sein.

Die Bewegung solcher unbemannter Flugkörper am Boden ist überdies ein kompliziertes Thema, weshalb man UAVs kaum je auf belebten Flughäfen zu sehen bekommt. Ebenfalls noch ungeklärt ist die Beherrschung der Einführungsphase, wenn sich bemannte und unbemannte Fliegerei im Luftraum mischt. Realistisch scheint hingegen für einen überschaubaren Zeitraum die Vision eines computergesteuerten Verkehrsflugzeuges, bei dem im Cockpit ein Mann (sicher nicht zwei) für alle Fälle Wache hält und der Bodenleitstand im Fall des Falles die Kontrolle übernimmt.

\subsection{Folgen}

Beide Tendenzen, Aufkommen der Very Light Jets auf kurze Sicht und die Automatisierung im Cockpit der Verkehrsflugzeuge in längerer Perspektive, bedeuten für junge Piloten, dass sich ihre berufliche Zukunft verdunkelt. Der Bedarf an qualifizierten Flugzeugführern steigt in diesem Szenario nicht mehr automatisch mit dem Wachstum des Luftverkehrs an - im Gegenteil. Der Traumberuf »echter Jungs« war früher Lokomotivführer und später Lastwagenfahrer auf der Langstrecke als »Kapitän der Landstraße« - ein durch die Realität längst entzauberter Mythos. Der Traumberuf Pilot könnte in absehbarer Zeit einen vergleichbaren Entzauberungseffekt erfahren.

\section{Ausblick}

Wir wissen, dass große Innovationen in aller Regel auf einer Zusammenfügung von zwei bereits bekannten Technologien basieren, die in isolierter Anwendung nur geringen Nutzen in einer kleinen Nische entfalten, aber gemeinsam einen markanten Durchbruch erlauben. Die gute alte Eisenbahn zum Beispiel verdankt ihren Erfolg einer Kombination der im Bergbau seit 500 Jahren bekannten Rad-Schiene Technik mit der im 18. Jahrhundert erfundenen Dampfmaschine (Biermann/ Dehr 1997).

Die Kombination der Very Light Jets und der UAVTechnologie, so lässt sich vorhersehen, bildet den logischen nächsten Schritt in der Entwicklung der Flugreise. Manager in der Mitte des 21. Jahrhunderts werden über einen Mini-Jet (beziehungsweise für kürzere Routen eine Art Hubschrauber) nicht weit vom Wohn- oder Arbeitsplatz verfügen, bei dem der Passagier selbst sein 
Ziel eintippt und auf Start drückt. Ohne menschlichen Piloten wird er/sie zum Ziel gebracht. Sollte ein Störfall eintreten, betätigt der Passagier einen Notknopf, so dass das Gerät automatisch zum nächsten Landeplatz fliegt oder sich eine Bodenzentrale zuschaltet, die manuell die Steuerung übernimmt.

Die Idee eines pilotenlosen Kleinflugzeuges gewinnt ihren besonderen Reiz im Fractional-Ownership-Modell bzw. in der Betriebsform als Luft-Taxi. So muss die Maschine nicht tagelang ungenutzt herumstehen, bis ihr Besitzer sie benötigt, sie könnte per Knopfdruck angefordert werden und nach dem Flugeinsatz gleich weiterreisen zum nächsten Nutzer. Positionierungsflüge ohne Passagiere sind der Alptraum jedes Flugplaners - sie verlieren ihren Schrecken, wenn kein Pilot mehr während solcher nicht-wertschöpfenden Tätigkeiten Geld kostet und möglicherweise just dann, wenn er gebraucht wird, aus der Ruhezeit fällt.

Aus ökonomischer Sicht bildet eine Kombination von VLJ und UAV deshalb eine durchaus überzeugende Vision, selbst wenn ein Einsatz in Mitteleuropa eher schwierig vorstellbar ist. Ein gutes Straßennetz, viele Schienenverbindungen und ein dicht bevölkerter Luftraum lassen hier für diese neuartigen Luftfahrzeuge kaum dringenden Bedarf erkennen. In China, Russland, Indien oder Brasilien hingegen überwiegen die Chancen ganz eindeutig die Probleme und Risiken. Aller Voraussicht nach wird diese neue Technologie zuerst in den USA in die Praxis gehen, weil dort das notwendige Know-how, die Kapitalkraft und die Innovationsfreude gebündelt vorhanden sind und vor allem in der Fläche abseits der Metropolregionen mit großer Nachfrage zu rechnen ist.

Dieser Beitrag basiert auf Erkenntnissen aus der Diskussionsveranstaltung »Captain Computer - Chancen und Risiken durch Automatisierung im Luftverkehr «, durchgeführt vom Wildau Institute of Technology (WIT) und dem TÜV Nord im Mai 2007 in Berlin unter Beteiligung von Experten der Berliner Flughäfen, des DLR (Deutsches Zentrum für Luft und Raumfahrt), der DFS (Deutsche Flugsicherung), der Lufthansa und des UAV D·A.CH (Unmanned Aerial Vehicle Working Group).

\section{Literatur}

Biermann, Thomas, Yield management in the airline Industry, Vortrag auf der LINDI-Konferenz, Wildau, September 2007

Biermann, Thomas, Gehört der Himmel den ALDI-Airlines? Neuer Wettbewerb im europäischen Luftverkehr, in: Wissenschaftliche Beiträge der TFH Wildau 2005

Biermann, Thomas/Dehr, Gunter (Hrsg.), Innovation mit System, Berlin, Heidelberg 1997

Cox, T./Nagy, C./Skoog, M./Somers, I., Civil UAV Capability Assessment, (Draft for NASA Directorate), 2004

Delfmann, Werner (Hrsg.), Strategic Management in the Airline Industry, Ashgate 2005

Shaw, Steven, Airline Marketing and Management, Ashgate 2004

Singh, Rajiv, VLJs - The rise of the hub busters, www.domain-b.com, 2006

Taneja, Nawal, Simply-flying - Optimizing the Airline Business Model, Ashgate 2004

The Economist, Fly me home James - travelling in an executive jet is just the ticket, 14. July 2007

Thompson, David, International Aviation's role in Productivity Growth and Climate Change - Can we resolve the dilemma? Vortrag Hamburg Aviation Conference, Februar 2007

Tretheway, Mike, Perspectives and Retrospectives, Vortrag Hamburg Aviation Conference, Februar 2007

\section{Internetquellen}

www.dlr.de

www.shephard.co.uk/UVonline

www.uavdach.org

\section{Autor}

Prof. Dr. rer. pol. Thomas Biermann

Wildau Institute of Technology

an der Technischen Fachhochschule Wildau

Tel. +49 3375 508-601

biermann@wit-wildau.de

www.wit-wildau.de 